\title{
Fluorescence Imaging Agent IC2000
}

National Cancer Institute

\section{Source}

National Cancer Institute. Fluorescence Imaging Agent IC2000. NCI Thesaurus. Code C151939.

A fluorescent imaging agent that can potentially be used as a diagnostic imaging agent. 